\title{
COVID-19: unravelling the clinical progression of nature's virtually perfect biological weapon
}

\author{
Giuseppe Lippi ${ }^{1}$, Fabian Sanchis-Gomar ${ }^{2,3 \#}$, Brandon M. Henry ${ }^{4 \#}$ \\ ${ }^{1}$ Section of Clinical Biochemistry, University of Verona, Verona, Italy; ${ }^{2}$ Department of Physiology, Faculty of Medicine, University of Valencia and \\ INCLIVA Biomedical Research Institute, Valencia, Spain; ${ }^{3}$ Division of Cardiovascular Medicine, Stanford University School of Medicine, Stanford, \\ California, USA; ${ }^{4}$ Cardiac Intensive Care Unit, The Heart Institute, Cincinnati Children's Hospital Medical Center, Ohio, USA \\ Contributions: (I) Conception and design: G Lippi; (II) Administrative support: None; (III) Provision of study materials or patients: None; (IV) \\ Collection and assembly of data: All authors; (V) Data analysis and interpretation: All Authors; (VI) Manuscript writing: All authors; (VII) Final \\ approval of manuscript: All authors. \\ "These authors share senior authorship. \\ Correspondence to: Prof. Giuseppe Lippi. Section of Clinical Biochemistry, University Hospital of Verona, Piazzale LA Scuro, 37134 Verona, Italy. \\ Email: giuseppe.lippi@univr.it
}

\begin{abstract}
Coronavirus disease 2019 (COVID-19) pandemic has shocked the world and caused morbidity and mortality on an unprecedented level in the era of modern medicine. Evidence generated to-date on the virulence and pathogenicity of severe acute respiratory syndrome coronavirus 2 (SARS-CoV-2) suggests that COVID-19 may be considered a perfect storm, caused by a nature's virtually perfect biological weapon. This conclusion is supported by an updated analysis of pathogenesis and clinical progression of this infectious disease. It is now readily apparent that COVID-19 is not a clear-cut disorder, but is instead a gradually evolving pathology, characterized by a series of stages sustained by different molecular and biological mechanisms. The disease can hence be divided in at least five different phases (incubation, respiratory, proinflammatory, pro-thrombotic, and death or remission). Whilst the virus triggers direct cytopathic injury during the initial stage of illness, in the following evolving phases, it is the host itself that undergoes an almost suicidal reaction, sustained, amplified and maintained by the immune, complement and hemostatic systems. Another peculiar property making SARS-CoV-2 a devious and vicious pathogen is the biophysical structure of its receptor biding domain, which needs to be primed by human proteases, thus being less efficiently targetable by the host immune system. The unique pathophysiology of COVID-19 requires the customization of therapy by individual patient characteristics and according to the phase-specific, evolving derangement of the multiple biological pathways.
\end{abstract}

Keywords: Coronavirus disease 2019 (COVID-19); coronavirus; pathogenesis; inflammation; coagulation

Submitted May 15, 2020. Accepted for publication May 30, 2020.

doi: 10.21037/atm-20-3989

View this article at: http://dx.doi.org/10.21037/atm-20-3989

\section{Introduction}

Coronavirus disease 2019 (COVID-19) pandemic has shocked the world and caused morbidity and mortality on an unprecedented level in the era of modern medicine. Caused by severe acute respiratory syndrome coronavirus 2 (SARS-CoV-2), this pathology has already affected millions of people worldwide, causing nearly four hundred thousand deaths to-date (1). Similar to the other two previous coronavirus outbreaks, severe acute respiratory syndrome (SARS) in 2002 to 2003 and Middle-East respiratory syndrome in 2012, COVID-19 may be characterized by a rather unfavorable, often critical, clinical progression. The global death rate of COVID-19 is now approaching $7 \%$ according to the regularly updated statistics from the World Health Organization (WHO) (1), with some notable 


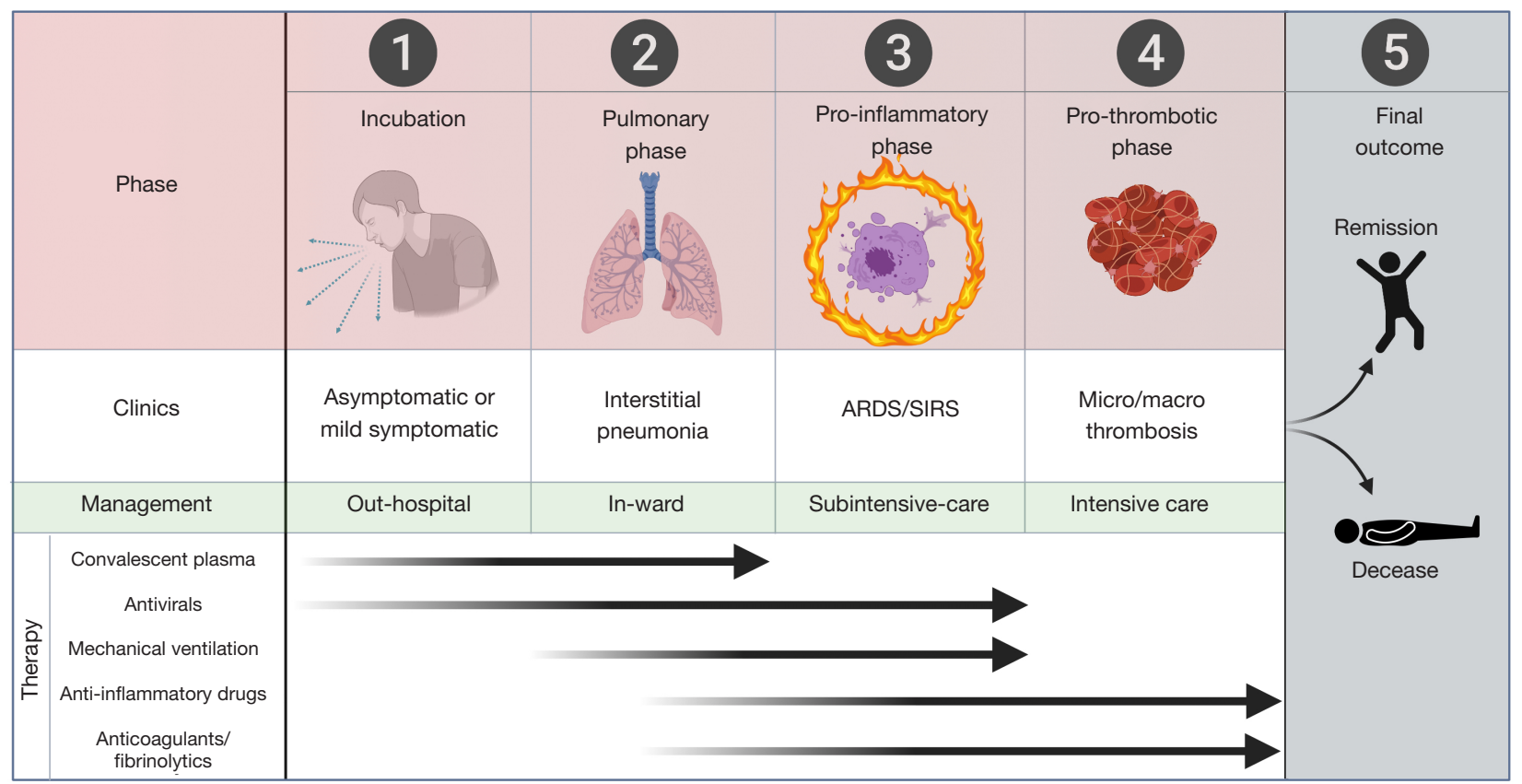

Figure 1 Phases, clinical progression, management and available therapies of COVID-19. ARDS, acute respiratory distress syndrome; SIRS, severe inflammatory response syndrome.

outliers (e.g., as high as $13.8 \%$ in Italy) due to the influence of some demographic, clinical and environmental factors impacting disease progression (2). Based on recent evidence on the virulence and pathogenicity of SARS-CoV-2, the clinical progression of COVID-19 can be divided into as many as five different phases (Figure 1). In the following sections of this article, we will describe the different characteristics of each phase of COVID-19.

\section{Phase 1: incubation}

This is the very first phase, which starts from infection up to the first onset of severe symptoms and usually lasts between 2 and 11 days (mean incubation time: 6 days), with patients likely to be infectious 1-3 days before symptom onset (3). Although the true rate of individuals who will remain asymptomatic, or only mildly symptomatic, until terminal viral shedding is still unknown, some evidence suggests that the number could be as high as $50 \%$ (4). Importantly, this rate may be even underestimated due to under-testing or under-reporting (4). It seems now reasonable to hypothesize that this pre-symptomatic phase is perhaps the most critical for containing the outbreak. Interestingly, the viral load of asymptomatic, presymptomatic, or mildly symptomatic subjects is comparable to that of patients with overt disease (5). This highlights the significant risk of viral transmission throughout this first phase. Evidence suggests that $50-80 \%$ of all cases may be attributed to transmission from an asymptomatic or pre-symptomatic individual (6). As such, the relatively long incubation time and the considerably high rate of asymptomatic-paucisymptomatic individuals combine to explain the pandemic and the rise in cases despite public health intervention and public awareness.

\section{Phase 2: progressive respiratory involvement}

The second phase, which involves $\sim 30 \%$ of all SARSCoV-2 infected subjects (7-9), is characterized by the onset of the typical COVID-19 symptoms. These symptoms basically include, but are not limited to, fever in $~ 85 \%$ of all patients, dyspnea ( 70-80\%), cough ( 40-80\%), olfactory and gustatory dysfunction $(\sim 50 \%)$, myalgia $(\sim 30 \%)$, gastrointestinal symptoms $(\sim 10-30 \%)(7-9)$. The typical symptoms of upper respiratory infections such as sore throat, nasal congestion and rhinorrhea are only present in a minority of patients $(<20 \%)(7-9)$. The high prevalence of dyspnea in these patients reflects the progressively severe respiratory involvement, which is variably associated with chest computed tomography imaging with ground-glass 
opacities and mixed consolidation, pleura and interlobular septal thickening, and air bronchograms (10). Interestingly, silent hypoxemia may occur, especially in the elderly, characterized significant hypoxemia and respiratory failure without overt signs of respiratory distress (11).

\section{Phase 3: focal and systemic inflammation}

The third phase, which develops in around $15 \%$ of all SARS-CoV-2 infected subjects (7-9), is perhaps the most challenging and intriguing from a physiopathological perspective. In fact, whilst the respiratory phase is mostly attributable to direct cytopathic lung injury caused by viral replication in pulmonary parenchyma, the pro-inflammatory phase is instead characterized by an abnormal, almost exaggerated, host reaction against the pathogen, either locally (i.e., in the lung) or systemically, thus mimicking the pathogenesis of severe sepsis and severe inflammatory response syndrome (SIRS) (12). Although the precise mechanisms underlying the onset of this disproportionate host response against the virus remain partially elusive, it has now been acknowledged that SARS-CoV-2 infection of dendritic cells and cells of the monocyte/macrophage lineage triggers their activation and active secretion of a vast array of pro-inflammatory cytokines such as interleukin (IL)-6, IL-2, IL-7, monocyte chemoattractant protein 1 (MCP-1), macrophage inflammatory protein 1- $\alpha$ (MIP $1-\alpha$ ), granulocyte colony stimulating factor (GSF), C-X-C motif chemokine 10 (CXCL10) and tumor necrosis factor- $\alpha$ $(\mathrm{TNF}-\alpha)(13,14)$. The renin-angiotensin-aldosterone system (RAAS) plays also a very relevant role in this phase. The binding of SARS-CoV-2 to its receptor angiotensinconverting enzyme 2 (ACE2) at the surface of host cells may be associated with profound derangement of RAAS, culminating in the increased activity of angiotensin II (Ang II) and decreased activity of angiotensin 1,7 (Ang 1,7), thus fostering vasoconstrictive, inflammatory, oxidative and fibrotic injuries (15).

The interplay between the virus and the host immune system may be even more complicated as evidence suggests that SARS-CoV-2 is capable of infecting CD4+ and CD8+ $\mathrm{T}$ lymphocytes, thus producing direct cytopathic effects and further contributing to generate a completely dysregulated cytokine pattern (16). Importantly, evolving lymphopenia may also be associated with a defect in antiviral and immune regulatory immunity (17). This highly abnormal immunoinflammatory response has now been universally defined as "cytokine storm" and is accompanied by a variety of focal injuries in multiple organs and tissues. More specifically, lung histopathology reveals the presence of activated pneumocytes, capillary congestion, inflammatory infiltrates, endothelial injury, protein-enriched interstitial edema and diffuse alveolar damage, a clear picture reflecting the development of acute respiratory distress syndrome (ARDS) (18). Notably, at this stage of disease, some typical cytopathic signs attributable to direct viral injury and inflammatory infiltrates can also be found in many other organs and tissues, such as the heart, kidney, liver, and intestine.

\section{Phase 4: microvascular and macrovascular thrombosis}

The fourth progressive stage of COVID-19, that appears cumulatively the most severe, tends to develop in around $5 \%$ of all SARS-CoV-2 infected subjects (7-9), and is characterized by the onset of microvascular and macrovascular thrombosis fostered by an exaggerated focal and/or systemic inflammation (19). The pro-thrombotic effects of hyperinflammation in veins and arteries are now widely recognized, and encompass a certain number of pathogenetic mechanisms (endothelial dysfunction, plaque instability, platelet hyper-activation, activation of complement system and blood coagulation, hypofibrinolysis), which have been comprehensively reviewed elsewhere $(20,21)$. The final result is the development of microvascular and macrovascular thrombosis, the former being virtually commonplace in all COVID-19 patients progressing to this fourth stage, and reflected evidence at lung histopathology of large inflammatory infiltration, microvascular thromboembolism and complete destruction of alveolar septae (18). In fact, it may be suspected that microvascular thrombosis begins early in the disease course and contributes significantly to lung injury leading to progression to severe illness. As such, appropriate anticoagulant or fibrinolytic therapy earlier in disease course may prevent progression to phase 4 .

Macrovascular complications can also be frequently observed in severely ill COVID-19 patients, manifesting as deep vein thrombosis (DVT), pulmonary embolism (PE), in situ pulmonary thrombosis and/or acute coronary syndrome (ACS) (22-25). Even in younger patients ( $<50$ years old), significant thrombotic events have been reported, including large vessel stroke (originating from carotid and cerebral arteries) (26). Notably, up to one-third of COVID-19 patients who die have evidence of pulmonary thrombosis as a 
direct cause of death (18). This matches clinical observations in which an incidence of thrombotic complications is reported to be $\sim 1 / 3^{\text {rd }}$ in critically ill COVID-19 patients, with the majority suffering from PE (25). In summary, COVID-19 induces a hypercoagulable state leading to micro- and macrovascular thrombi that significantly contribute to lung injury and multi-organ dysfunction in COVID-19 patients.

\section{Phase 5: death or remission}

The final stage of disease can evolve into two different outcomes, decease or remission. The current statistics of intensive care unit mortality are quite heterogeneous, with death rates varying between $20 \%$ and $80 \%$ (27), depending on multiple demographic, clinical and environmental factors. Death is mostly attributable to ARDS, pulmonary thrombosis, acute renal failure, acute cardiac injury, superinfection and/or multiple organ failure $(7,28,29)$.

\section{Beyond COVID-19 remission?}

After symptom resolution and successful recovery, questions remain over the intermediate and long-term health impacts of COVID-19. In one study, $94 \%$ of discharged patients had residual disease on their final CT scans, most commonly characterized by ground glass opacities (30). Whether COVID-19 leads to permanent lung scarring and fibrosis requires further investigation. Moreover, it may be suspected that COVID-19 induced tissue damage, such as cardiac or renal injury, may exacerbate pre-existing comorbidities, thus impacting long term health of patients.

In children, though the course of COVID-19 is mostly mild, a concerning new post-viral phenomenon has emerged in recent weeks and suspected to be related to SARS-CoV-2. Called multisystem inflammatory syndrome in children (MIS-C), it is described as a "hyperinflammatory shock" that presents with characteristics similar to Kawasaki disease and toxic shock syndrome (31). Suspected MIS-C has resulted in a concerning rise in COVID-19 related admissions to pediatric intensive care units in recent weeks, with several fatalities now reported. The emergence of this new phenomenon highlights how there is much still to be elucidated in the pathophysiology of this novel virus.

\section{A devious enemy}

Besides the complex and mechanistic interplay responsible for direct and indirect host injuries, SARS-CoV-2 has another peculiar property that makes it a devious and vicious pathogen; the biophysical structure of its spike (S)-protein receptor-binding domain (RBD) is highly ingenious. Unlike its homologous precursor, which caused the SARS outbreak and that has now been renamed SARS-CoV-1, the RBD of SARS-CoV-2 displays ineffective receptor binding at rest (32) because it needs to be primed by human proteases such as the transmembrane serine protease 2 (TMPRSS2) or furin $(32,33)$. The $\mathrm{S} 1$ subunit on the S-protein RBD is dissociated from the $\mathrm{S} 2$, which facilitates the fusion with the host cell membrane (34). Overall, the RDB of SARS-CoV-2 seems to be less efficiently targetable by the immune system, which may struggle to arrange an efficient immune response against the virus. This theory is confirmed by evidence that the process of antibody generation in the course of SARS$\mathrm{CoV}-2$ infection is atypical and unique. IgM, the first class of immunoglobulins that typically appears in response to infectious pathogens, may be absent in $20-40 \%$ of SARS$\mathrm{CoV}-2$ infected individuals (35), has a typically low titer $(36,37)$, and, importantly, does not specifically target the S-protein RBD, which makes it theoretically ineffective at neutralizing the virus. The capacity to eradicate the infection may hence be delayed until a more specific, robust, and permanent immune response will emerge, specifically encompassing the generation of $\operatorname{IgG}$ immunoglobulins (38). Finally, it remains unknown whether long term protective immunity is afforded post-recovery by antibody production and immunological memory.

\section{Conclusions}

Irrespective of the origin and intermediate hosts of SARS-CoV-2 (39), the evidence garnered to-date on its virulence and pathogenicity would lead us to conclude that COVID-19 is the portrait of a perfect storm (40) caused by nature's virtually perfect biological (lethal) weapon. Whilst in the initial stage of illness the virus causes direct cytopathic injury, in the following evolving phases, it is the host itself that undergoes an almost suicidal reaction, sustained, amplified and maintained by the immune, complement and hemostatic systems. It is now readily apparent that COVID-19 is not a clear-cut disorder, but is instead a gradually evolving pathology characterized by a series of stages sustained by different molecular and biological mechanisms, whose understanding is essential for establishing the most appropriate, phase-specific treatment. COVID-19 would appear as a paradigm example 
of personalized medicine, whereby the therapy needs to be tailored not only to individual characteristics (e.g., male sex, older age, presence of co-morbidities), but also according to the evolving derangement of multiple biological pathways.

\section{Acknowledgments}

Funding: None.

\section{Footnote}

Conflicts of Interest: All authors have completed the ICMJE uniform disclosure form (available at http://dx.doi. org/10.21037/atm-20-3989). GL serves as an unpaid executive editor-in-chief of Annals of Translational Medicine from Jan 2016 to Jan 2022. FSG serves as an unpaid editorial board member of Annals of Translational Medicine from Mar 2019 to Feb 2021. BMH has no conflicts of interest to declare.

Ethical Statement: The authors are accountable for all aspects of the work in ensuring that questions related to the accuracy or integrity of any part of the work are appropriately investigated and resolved.

Open Access Statement: This is an Open Access article distributed in accordance with the Creative Commons Attribution-NonCommercial-NoDerivs 4.0 International License (CC BY-NC-ND 4.0), which permits the noncommercial replication and distribution of the article with the strict proviso that no changes or edits are made and the original work is properly cited (including links to both the formal publication through the relevant DOI and the license). See: https://creativecommons.org/licenses/by-nc-nd/4.0/.

\section{References}

1. World Health Organization. Coronavirus disease (COVID-2019) situation reports. Available online: https://www.who.int/emergencies/diseases/novelcoronavirus-2019/situation-reports/

2. Lippi G, Mattiuzzi C, Sanchis-Gomar F, et al. Clinical and demographic characteristics of patients dying from COVID-19 in Italy versus China. J Med Virol 2020. doi: 10.1002/jmv. 25860.

3. Lai CC, Shih TP, Ko WC, et al. Severe acute respiratory syndrome coronavirus 2 (SARS-CoV-2) and coronavirus disease-2019 (COVID-19): The epidemic and the challenges. Int J Antimicrob Agents 2020;55:105924.

4. Istituto Superiore di Sanità. Integrated surveillance of COVID-19 in Italy. Available online: https:// www.epicentro.iss.it/en/coronavirus/bollettino/ Infografica_11maggio\%20ENG.pdf

5. Zou L, Ruan F, Huang M, et al. SARS-CoV-2 Viral Load in Upper Respiratory Specimens of Infected Patients. N Engl J Med 2020;382:1177-9.

6. Gandhi RT, Lynch JB, Del Rio C. Mild or Moderate Covid-19. N Engl J Med 2020. doi: 10.1056/ NEJMcp2009249.

7. Istituto Superiore di Sanità. Characteristics of SARS$\mathrm{CoV}-2$ patients dying in Italy. Report based on available data on May 7th, 2020. Available online: https://www. epicentro.iss.it/en/coronavirus/bollettino/ReportCOVID-2019_7_may_2020.pdf

8. Garg S, Kim L, Whitaker M, et al. Hospitalization Rates and Characteristics of Patients Hospitalized with Laboratory-Confirmed Coronavirus Disease 2019 COVID-NET, 14 States, March 1-30, 2020. MMWR Morb Mortal Wkly Rep 2020;69:458-64.

9. Tong JY, Wong A, Zhu D, et al. The Prevalence of Olfactory and Gustatory Dysfunction in COVID-19 Patients: A Systematic Review and Metaanalysis. Otolaryngol Head Neck Surg 2020. doi: 10.1177/0194599820926473.

10. Bao C, Liu X, Zhang H, et al. Coronavirus Disease 2019 (COVID-19) CT Findings: A Systematic Review and Meta-analysis. J Am Coll Radiol 2020. doi: 10.1016/ j.jacr.2020.03.006.

11. Xie J, Tong Z, Guan X, et al. Critical care crisis and some recommendations during the COVID-19 epidemic in China. Intensive Care Med 2020;46:837-40.

12. Singer M, Deutschman CS, Seymour CW, et al. The Third International Consensus Definitions for Sepsis and Septic Shock (Sepsis-3). JAMA 2016;315:801-10.

13. Moore JB, June CH. Cytokine release syndrome in severe COVID-19. Science 2020;368:473-4.

14. Mehta P, McAuley DF, Brown M, et al. COVID-19: consider cytokine storm syndromes and immunosuppression. Lancet 2020;395:1033-4.

15. Henry BM, Vikse J, Benoit S, et al. Hyperinflammation and derangement of renin-angiotensin-aldosterone system in COVID-19: A novel hypothesis for clinically suspected hypercoagulopathy and microvascular immunothrombosis. Clin Chim Acta 2020;507:167-73.

16. Tan L, Wang Q, Zhang D, et al. Lymphopenia predicts disease severity of COVID-19: a descriptive and predictive 
study. Signal Transduct Target Ther 2020;5:33.

17. Azkur AK, Akdis M, Azkur D, et al. Immune response to SARS-CoV-2 and mechanisms of immunopathological changes in COVID-19. Allergy 2020. doi: 10.1111/all.14364.

18. Wichmann D, Sperhake JP, Lutgehetmann M, et al. Autopsy Findings and Venous Thromboembolism in Patients With COVID-19: A Prospective Cohort Study. Ann Intern Med 2020. doi: 10.7326/M20-2003.

19. Jose RJ, Manuel A. COVID-19 cytokine storm: the interplay between inflammation and coagulation. Lancet Respir Med 2020. doi: 10.1016/S2213-2600(20)30216-2.

20. Bikdeli B, Madhavan MV, Jimenez D, et al. COVID-19 and Thrombotic or Thromboembolic Disease: Implications for Prevention, Antithrombotic Therapy, and Follow-up. J Am Coll Cardiol 2020. doi: 10.1016/ j.jacc.2020.04.031.

21. Willyard C. Coronavirus blood-clot mystery intensifies. Nature 2020. doi: 10.1038/d41586-020-01403-8.

22. Lodigiani C, Iapichino G, Carenzo L, et al. Venous and arterial thromboembolic complications in COVID-19 patients admitted to an academic hospital in Milan, Italy. Thromb Res 2020;191:9-14.

23. Poissy J, Goutay J, Caplan M, et al. Pulmonary Embolism in COVID-19 Patients: Awareness of an Increased Prevalence. Circulation 2020. doi: 10.1161/ CIRCULATIONAHA.120.047430.

24. Guzik TJ, Mohiddin SA, Dimarco A, et al. COVID-19 and the cardiovascular system: implications for risk assessment, diagnosis, and treatment options. Cardiovasc Res 2020. doi: 10.1093/cvr/cvaa106.

25. Klok FA, Kruip M, van der Meer NJM, et al. Incidence of thrombotic complications in critically ill ICU patients with COVID-19. Thromb Res 2020. doi: 10.1016/ j.thromres.2020.04.013.

26. Oxley TJ, Mocco J, Majidi S, et al. Large-Vessel Stroke as a Presenting Feature of Covid-19 in the Young. N Engl J Med 2020;382:e60.

27. Halacli B, Kaya A, Topeli A. Critically-ill COVID-19 patient. Turk J Med Sci 2020;50:585-91.

28. Vincent JL, Taccone FS. Understanding pathways to death in patients with COVID-19. Lancet Respir Med 2020;8:430-2.

Cite this article as: Lippi G, Sanchis-Gomar F, Henry BM. COVID-19: unravelling the clinical progression of nature's virtually perfect biological weapon. Ann Transl Med 2020;8(11):693. doi: 10.21037/atm-20-3989
29. Grasselli G, Zangrillo A, Zanella A, et al. Baseline Characteristics and Outcomes of 1591 Patients Infected With SARS-CoV-2 Admitted to ICUs of the Lombardy Region, Italy. JAMA 2020;323:1574-81.

30. Wang Y, Dong C, Hu Y, et al. Temporal Changes of CT Findings in 90 Patients with COVID-19 Pneumonia: A Longitudinal Study. Radiology 2020. doi: 10.1148/ radiol.2020200843.

31. Riphagen S, Gomez X, Gonzalez-Martinez C, et al. Hyperinflammatory shock in children during COVID-19 pandemic. Lancet 2020. doi: 10.1016/S01406736(20)31094-1.

32. Walls AC, Park YJ, Tortorici MA, et al. Structure, Function, and Antigenicity of the SARS-CoV-2 Spike Glycoprotein. Cell 2020;181:281-92.e6.

33. Shang J, Wan Y, Luo C, et al. Cell entry mechanisms of SARS-CoV-2. Proc Natl Acad Sci U S A 2020. doi: 10.1073/pnas.2003138117.

34. Belouzard S, Millet JK, Licitra BN, et al. Mechanisms of coronavirus cell entry mediated by the viral spike protein. Viruses 2012;4:1011-33.

35. Lippi G, Salvagno GL, Pegoraro M, et al. Assessment of immune response to SARS-CoV-2 with fully automated MAGLUMI 2019-nCoV IgG and IgM chemiluminescence immunoassays. Clin Chem Lab Med 2020. doi: 10.1515/ cclm-2020-0473.

36. Du Z, Zhu F, Guo F, et al. Detection of antibodies against SARS-CoV-2 in patients with COVID-19. J Med Virol 2020. doi: $10.1002 /$ jmv. 25820.

37. Qu J, Wu C, Li X, et al. Profile of IgG and IgM antibodies against severe acute respiratory syndrome coronavirus 2 (SARS-CoV-2). Clin Infect Dis 2020. doi: 10.1093/cid/ ciaa489.

38. Zhang Y, Xu J, Jia R, et al. Protective humoral immunity in SARS-CoV-2 infected pediatric patients. Cell $\mathrm{Mol}$ Immunol 2020. doi: 10.1038/s41423-020-0438-3.

39. Lippi G, Plebani M. The novel coronavirus (2019-nCoV) outbreak: think the unthinkable and be prepared to face the challenge. Diagnosis (Berl) 2020;7:79-81.

40. Lippi G, Sanchis-Gomar F, Henry BM. Coronavirus disease 2019 (COVID-19): the portrait of a perfect storm. Ann Transl Med 2020;8:497. 